\title{
Construction of Chinese University Culture from the Perspective of Multimodal Metaphor and Metonymy-A Case Study on the 2018 Admissions Film 'Graduation Answers' of Tsinghua University
}

\author{
Jin Huan ${ }^{1}$ \& Duan Youguo ${ }^{2 \#}$ \\ School of Foreign Studies, Yangtze University, Hubei, 434023 P.R. China.
}

\#corresponding author.

Type of Work: Peer-Reviewed

DOl: http://dx.doi.org/10.21013/jems.v15.n5.p5

\section{How to cite this paper:}

Huan, J., Youguo, D. (2019). Construction of Chinese University Culture from the Perspective of Multimodal Metaphor and Metonymy-A Case Study on the 2018 Admissions Film 'Graduation Answers' of Tsinghua University. IRA International Journal of Education and Multidisciplinary Studies (ISSN 2455-2526), 15(5), 191-200.doi: http://dx.doi.org/10.21013/jems.v15.n5.p5

(C) Institute of Research Advances.

This work is licensed under a Creative Commons Attribution-Non Commercial 4.0 International License subject to a proper citation to the publication source of the work.

Disclaimer: The scholarly papers as reviewed and published by the Institute of Research Advances (IRA) are the views and opinions of their respective authors and are not the views or opinions of the IRA. The IRA disclaims of any harm or loss caused due to the published content to any party.

Institute of Research Advances is an institutional publisher member of Publishers International Linking Association Inc. (PILA-CrossRef), USA. The institute is an institutional signatory to the Budapest Open Access Initiative, Hungary advocating the open-access of scientific and scholarly knowledge. The Institute is a registered content provider under Open Access Initiative Protocol for Metadata Harvesting (OAI-PMH).

The journal is indexed \& included in WorldCat Discovery Service (USA), CrossRef Metadata Search (USA), WorldCat (USA), OCLC (USA), Open J-Gate (India), EZB (Germany) Scilit (Switzerland), Airiti (China), Bielefeld Academic Search Engine (BASE) of Bielefeld University, Germany, PKP Index of Simon Fraser University, Canada.

\footnotetext{
1 Jin Huan is a postgraduate of QX181 in the School of Foreign Studies, Yangtze University. ${ }^{2}$ Duan Youguo (corresponding author) is an Associate Professor in the School of Foreign Studies, Yangtze University. He is interested in cognitive linguistics.
} 


\section{ABSTRACT}

The admissions films of the university are an important way to show their culture to the outside world, which is a typical multimodal discourse. By analyzing the construction of university culture in the "Graduation Answers" from the perspective of multimodal metaphor and metonymy, it is found that there are multi-modal metaphors such as images and words, gestures and words, music and words, and metonymies such as individual representing entirety and clothing representing identity in the admission film, which enhance the expressive power of the video and resonate with the audience.

Keywords: University admissions film; University culture; Multimodal metaphor; Multimodal metonymy

\section{Introduction}

Lakoff and Johnson (1980) pointed out that metaphor is a way of thinking and behaving, and language is just the external form of conceptual metaphor. The academic community has generally recognized the thinking and conceptual nature of metaphor at present. However, with the development of science and technology, scholars have found that in addition to language, people can also communicate with each other by other communicative symbols, such as sound, color, lines, spatial layout, etc. (Forceville, 2002, 2006, 2008). Thus, in the 1990s, a group of scholars led by Forceville extended the conceptual metaphor of linguistics to the field of multimodal research. And the collection of Multi-modality Metaphor edited and published by Forceville\&Urios-Aparisi in 2009 marks the formal formation of multimodal metaphors (Zhao Xiufeng, 2011). Multimodal metaphors focus on the earliest and most common categories of advertisements (Zhao Xiufeng, 2011), like print ads and dynamic ads. Some scholars in China explore the construction of multimodal metaphors and metonymy in public service advertisements (Guan Ke, 2015; Han Yafeng, 2015; Pan Hongying, 2013, etc.).And the other scholars analyze multimodal metaphors and metonymy in commercial advertisements. (Wang Yang \& Xiang Enbai, 2016; Zhang Hui \& Zhan Weiwei, 2011, etc.). Among varying video advertisements, there are few studies on multimodal metaphors and metonymy in college admissions video advertisements. The reason may be that college admissions advertisements are different from other commercial advertisements or public service advertisements. College admissions advertisements are not only a means of communication but also an important part of university culture (Dong Bo, 2019). Based on this, this paper selects Tsinghua University's enrollment propaganda film in 2018 as a corpus, and analyzes the propaganda film from the perspective of multimodal metaphor and metonymy, hoping to unearth the construction of Chinese college culture from the perspective of the multi-modal metaphor and metonymy, which will provide a new direction for future research on admissions videos.

\section{The Theoretical Basis}

\subsection{The multimodal metaphor}

Cognitive linguistics believes that the essence of metaphor is to understand and experience another kind of thing through one kind of thing, which is the result of projecting from the source domain to the target domain (Lakoff \& Johnson, 1980). Multimodal metaphor refers to the emergence of the source and target domains of metaphor in different modalities (Forceville, 2006). Forceville (2008) has proposed three conditions for defining multimodal metaphors. First, the context in which the two phenomena are located belongs to different categories. Second, the two phenomena can be considered as the source domain and the target domain, respectively. Finally, the two phenomena are characterized by two or more symbology and sensory modalities. Zhao Xiufeng (2011) also shows that the essence of metaphor is based on the cross-domain mapping of similarity.For research convenience, Forceville(2006) subdivides the modality into: (1) image symbol; (2) written symbol; (3) verbal symbol; (4) gesture; (5) sound; (6) music; (7) smell; (8) taste; (9) contact.However, the definition of multimodal metaphor is 
difficult to grasp in practice. In a narrow sense, the source and target languages of multimodal metaphors are characterized by or mainly by different modalities. In a broad sense, multimodal metaphors are constructed by two or more modes. In general, the modality of the text must be included in the construction of one of the conceptual domains. The multimodality in college propaganda films mainly involves four modes such as text (narration and subtitles), images, gestures and music, so this paper adopts the concept of generalized multimodal metaphor, which will analyze the metaphors between images and words, gestures and words, music and words to explore the construction of college culture in the admissions film.

\subsection{The multimodal metonymy}

Metonymy is composed of mappings within the same empirical domain or conceptual structure (Lakoff \& Turner,2009). A generally accepted view is to use A to refer to B. There is no clear definition of multimodal metonymy in the academic community. But the multimodal metonymy can be defined as the initial source domain constructed by two or more modes of vision, hearing, and so on to start the target domain of the same cognitive domain referring to the concept of multimodal metaphor (Wang Xiaomei, 2019). This article mainly refers to the classification of metonymy by Peirsman and Geeraerts (2006). They have divided the metonymy into 6 modes : individual entity \& whole, subevent \& complex event, spatial part \& whole, antecedent \& consequent, location \& located and piece of clothing \& person. This paper will mainly analyze the two multimodal metonyms: individual entity \& whole and piece of clothing \& person. There are not many articles on the study of multimodal metonymy in the academic world and most of them use multimodal metonymy and metaphor to study together (Zhao Xiufeng et al., 2010; Yang Xu et al., 2013). There is a close connection between metaphor and metonymy. Metonymy is the premise of metaphor. The generation of metaphor is based on metonymy (Liu Zhengguang, 2002). The difference between them is that, first, metaphor is based on similarity mapping, while metonymy is based on interdependence; Second, metaphor is mapping cross-domain, but metonymy is in the same domain; Third, metaphor is based on metonymy, and metonymy can exist independently of metaphor. (Yan Jia, 2016)

\section{The introduction of Tsinghua university 2018 admissions film "Graduation Answers"}

Tsinghua University 2018 enrollment propaganda film "Graduation Answers" lasts 5 minutes and 33 seconds and consists of three parts, as shown in Table 1. The first part consists of six questions about students' life before Tsinghua University. The second part consists of seven questions before graduation. The last part is a part of the song Glass Wall.

\begin{tabular}{|c|c|}
\hline \multirow{4}{*}{ Part 1 } & Contents \\
\cline { 2 - 3 } & 1) Before you went to college, had you made an important decision in life? \\
\cline { 2 - 3 } & 2) Have you ever gone abroad and seen a bigger world? \\
\cline { 2 - 3 } & 3) Have your parents cultivated your hobbies? \\
\hline \multirow{4}{*}{ Part 2 } & 4) Do you know how to work with others? \\
\cline { 2 - 3 } & 5) Are you confident when you get along with others? \\
\cline { 2 - 3 } & 6) Do you influence others through your own efforts? \\
\cline { 2 - 3 } & 7) Have you walked out of the campus to explore a strange world? \\
\cline { 2 - 3 } & 9) Have you found what you love, do you love what you do? \\
\hline & 10) Have you done what you can't through cooperation? \\
\hline 11) Have you achieved self-improvement and strive to change your own destiny?
\end{tabular}


IRA-International Joumal of Education $\Xi^{\circ}$ Multidisciplinary Studies

\begin{tabular}{|c|c|}
\hline & 13) In Tsinghua, have you become a better self? \\
\hline Part 3 & A part of Glass Wall \\
\hline
\end{tabular}

Table1 : The components of the video

Liu Zhen, the director of the Admissions Office of Tsinghua University, invited 34 undergraduate graduates to participate in the answer sheet. The scene is located at an indoor stadium. There are radiant headlights in the center of the stadium, and there are six concentric circles with neon lights around the headlights (as shown in Figure 1). The examiner asks the questions and tells the students how to answer them, that is if the student's answer to the first six questions is "Yes", they needn't move; if the answer is "no", he or she need retreat to the next line. If the answer to the 7 questions in the second part is "No", they needn't move; if the answer is "Yes", he or she need go one step forward. The admission film mainly presents metaphors and metonymy through two of the most important information carriers, language and image. Van Leeuwen points out the relationship between images and language: interpreting and extending. The former interprets and refines the language through the image, or the language interprets the image and makes it concrete. The latter refers to the similarity of language and graphic content, which is further complemented by comparison (Van Leeuwen, 2005). In addition to this, gestures, expressions, music, etc. also complement multimodal metaphors and metonymy (Wang Xiaomei, 2019).

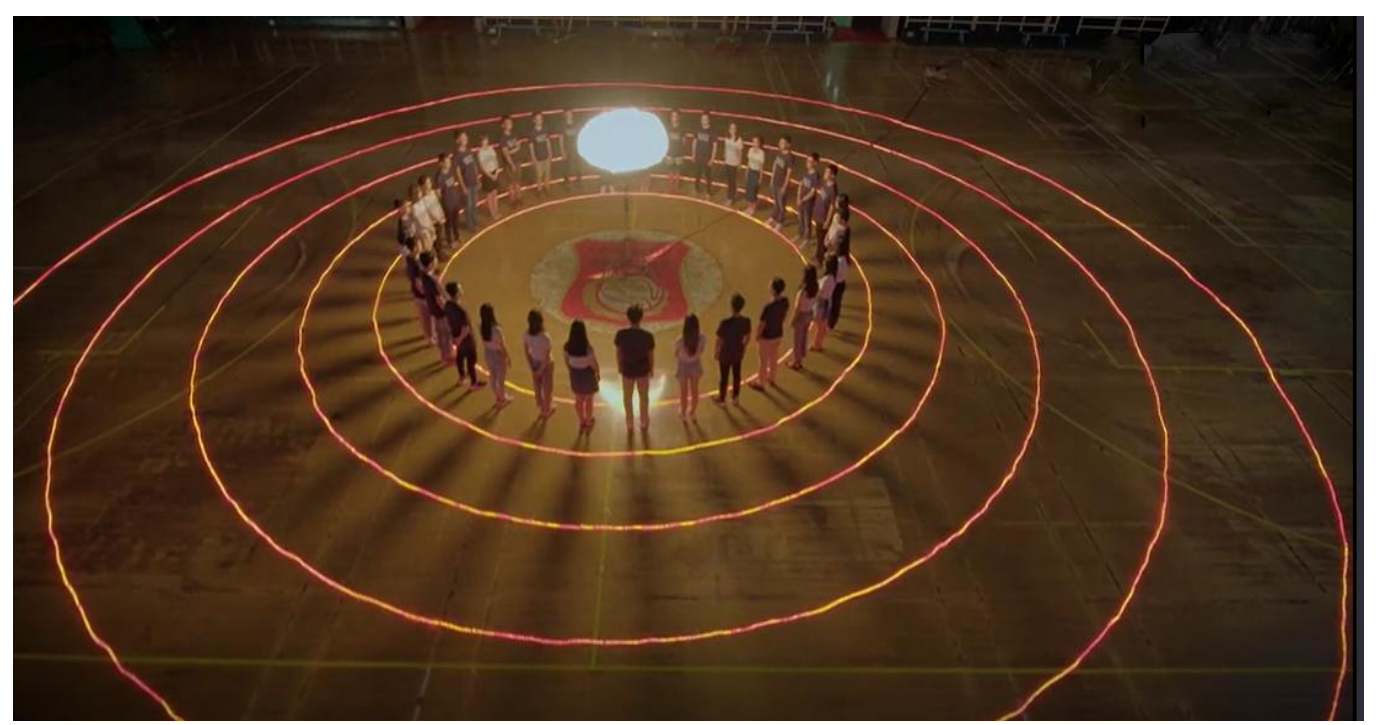

Figure 1: Scene layout

\section{The construction of multimodal metaphor in the Graduation Answers.}

Metaphors have the role of building bridges between the source domain and the target domain, helping readers or listeners better understand the speaker's intentions. Multimodal metaphors are more than a medium, multi-dimensional construction of the source domain to the target domain, in order to achieve better expression. The Graduation Answers uses the following multimodal metaphors to increase the appeal of the audience.

\subsection{Multimodal metaphor based on image and text}

Before starting the answer, 34 graduates stood on the same line, the same distance from the center. Such a position distribution of pictures means that everyone is equal in life. However, in answering the six questions before coming to Tsinghua, different students retreated to the five rings and six rings farther 
away from the center because of different problems (as shown in Figure 2). At this time, the picture metaphorizes the teacher's language "Four years ago, you entered Tsinghua. Different growth environments gave you the starting line when you entered Tsinghua." It also told the audience, before entering Tsinghua, the starting point of each student is different. That is to say, before entering Tsinghua University, students have already been different from the equal state of human life due to different growth environments and resources.

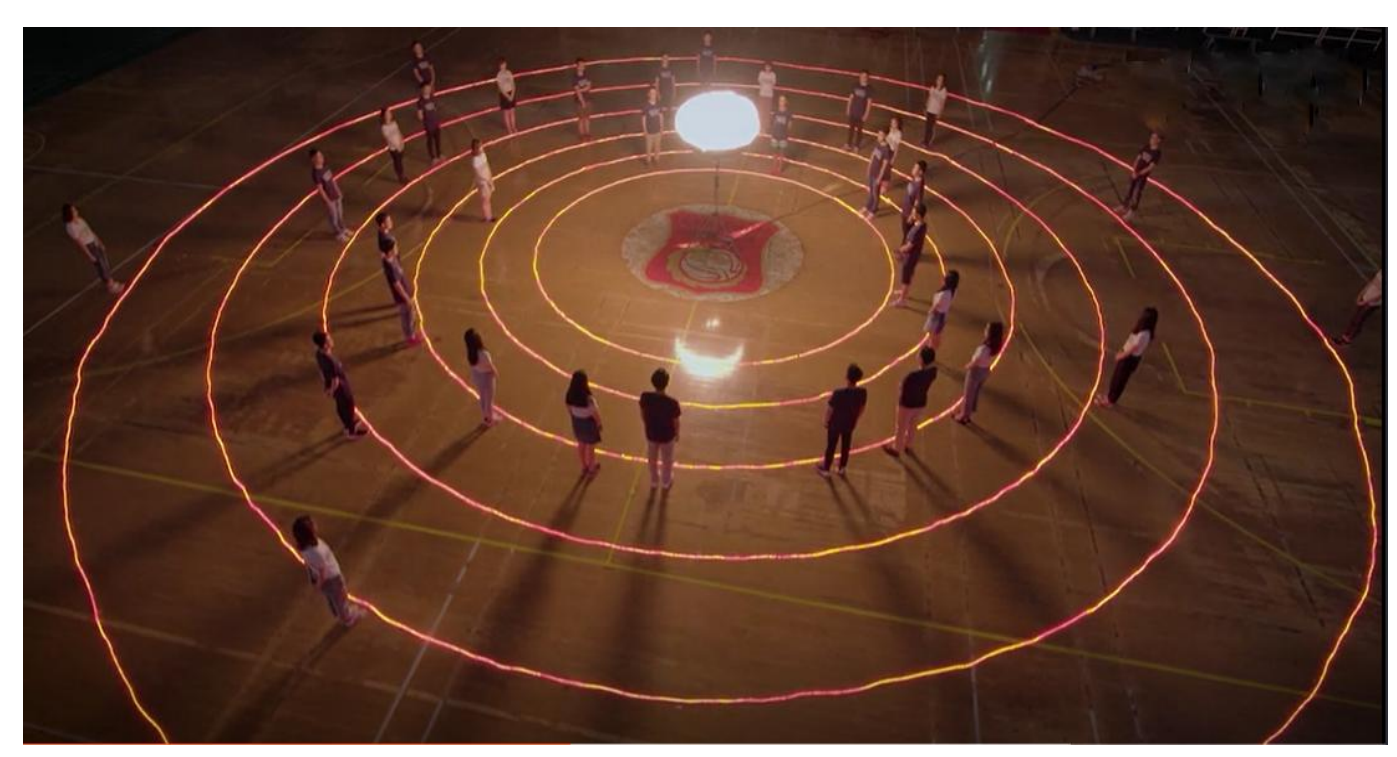

Figure 2: The life pattern before entering Tsinghua University

In the second part of the film, the examiner asked seven questions about the 34 undergraduates who are about to graduate. If the answers to these seven questions are yes, you need to move forward. Through the questions of these seven questions, the students at different starting lines have basically returned to the same starting line from the center of the stage (as shown in Figure 3). The camera shot directly from the top to the center of the stage and added "This is their answer sheet. This is Tsinghua's answer sheet." The position of the students in the text and video metaphorizes Tsinghua University's spirit of "Self-discipline and Social Commitment" which tells the audience no matter what your starting point is, as long as you work hard, have the confidence to change yourself, and maintain an open mind, you can achieve the same scores as other students who have different starting points, and thus gain the ability to change your own destiny. In the process, the radiant headlights in the center of the stadium are the colorful stage of life. Each student is in a different position in their life stage. Each question reflects the characteristics that a person needs in his life, such as decision-making (see questions $1 \& 7$ ), vision (see questions $2 \& 8$ ), interests (see questions $3 \& 9$ ), cooperative spirit (see questions $4 \& 10$ ), self-reliance (see questions $5 \& 11)$ and dedication (6 \& 12). 


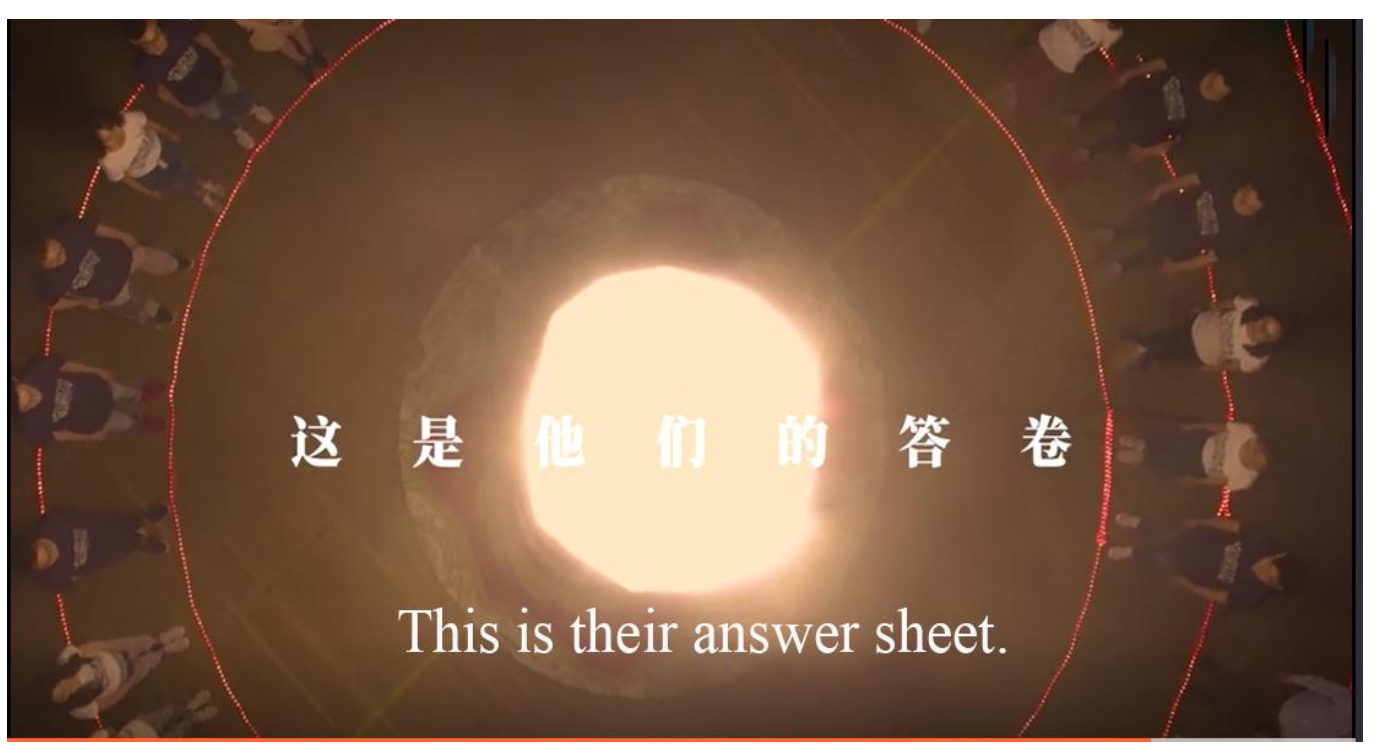

Figure 3: The life pattern of Tsinghua students who are about to graduate

\subsection{Multimodal metaphor based on gestures and words}

The Graduation Answers also uses a lot of gesture and words metaphors. In the first part of the video, some negative expressions and gestures are used to metaphorize the fact that most of the students are not perfect enough because of the growth environment or other reasons before coming to Tsinghua. However, in the second part of the video, more positive expression and gesture metaphors are used. The strong contrast with the previous expression metaphors metaphorize the growth and progress of students in Tsinghua during the four years. The multi-model metaphors based on gestures and words that appear in the video will be explained one by one.

In the first part of the video, when asked the first question (Before you went to college, had you made an important decision in your life?), a girl pouted, shook her head, lowered her head and stepped back. These expressions, together with the gestures metaphorized that she is in a state of low mood because she has not made important choices about life before going to college. When was asked the third question (Whether your parents have cultivated your hobbies?), a girl pouted and bowed back, which metaphorize that she had no hobbies before going to college. When was asked the third question (Whether your parents have cultivated your hobbies?), a girl pouted and bowed back, which metaphorizes that she had no hobbies before going to college. During the first student festival performance, the girl just watched others performing quietly under the stage. She wore a black T-shirt with the words "Going out, and run errands" printed on the front, followed by the words "Extra - we are not the same". From the choice of clothing, the girl expected to be able to have different talents from others, and can play with everyone in her spare time. However, because the girl has not been cultivated hobbies since childhood, she has to watch other students' performance at the time. The girl's envious smile and lost bow in the audience are all metaphors for girls' low mentality because she can't fit into others. When asked the fourth question (Do you know how to work with others?), a male student also pouted and retreated to the next line, recalling the scene of the "first freshman seminar" in which the boy played the mobile phone himself, remained silent during the discussion, and showed different opinion from other people in the final division of labor, which shows the boy does not know how to get along with others. When asked the fifth question (Are you confident when you get along with others?), a girl looks down and quickly recalls the situation when she first entered school. In this video, the girl wears black-rimmed glasses, a ponytail, and her eyes are looking forward. The hand pushes the 
middle of the frame and pulls the bangs behind her ears. When the seniors want to help her pick up her luggage, the girl instinctively refused and pulled the luggage back saying "No, no." The girl's expressions, gestures and words show the inferiority of the girl when she is with others.

In the second part of the video, when a girl was asked the seventh question (Do you have the courage to master your life now?), her memory switches to the past with the camera, closed the laptop and went out to call her mother and told that she would enroll in a PH.D. program. This series of actions, facial expressions and language metaphorize that the girl has the courage to master her life. When asked the eighth question (Have you walked out of the campus to explore a strange world?), a boy with a confident smile recalled his one-year study in Africa. When asked the ninth question (Do you find what you love, do you love what you do?), a boy lifted his head a little and recalled his experience of organizing more than 20 school-level activities during his undergraduate studies. When asked the tenth question (Do you complete what you can't do through cooperation?), a boy with a cane closes his lips and nods his head and recalls the experience that he was injured as a basketball coach to complete the final game of the undergraduate course. When asked the eleventh question (Have you achieved self-improvement and strive to change your own destiny?), the same girl who did not get along with others before, has now stood as a representative of outstanding undergraduates at the graduation ceremony and published a graduation testimonial. Finally, when asked the twelfth question (Are you willing to make things better and use the action to make the world better?), a student who will go to Hunan Jishou to teach after graduation appears in the video.

The strong contrast between the two parts of the gesture and expression metaphor highlights the students' growth in Tsinghua University. That is to say, before coming Tsinghua, they are inferior, narrow-minded, have no direction in life, lack cooperation, self-improvement and dedication. Through the four years of study at Tsinghua University, most students have found their own direction of life. Under the influence of Tsinghua's self-improvement and social commitment, students have learned to cooperate and become better themselves.

\subsection{Multimodal metaphor based on music and words}

The Graduation Answers also highlights and renders related themes through the background music. The contrast between the two parts of the music also reflects the growth of students in Tsinghua. In the first part of the video, the music is slow and quiet. With the following seven questions, the rhythm of the music speeds up and becomes excited until it comes out that "this is their answer sheet, and this is Tsinghua's answer sheet." With the dazzling stage center, the music turns to the ending song Glass Wall. This English song is accompanied by Chinese and English letters, which can better convey to the audience the purpose of changing destiny as long as they work hard. The change of the whole background music metaphorizes the student's transformation in Tsinghua, from the initial hesitation to the subsequent heroic decisiveness, from the previous ignorant inferiority to the later wisdom and self-confidence, from the previous narrow horizon to the present world vision. All these changes are accompanied by changes in music and are more deeply rooted in the hearts of the people.

\section{The construction of multimodal metonymy in Graduation Answers}

The source and target domains in metonymy often appear in the same cognitive domain, and the whole is referred to by the whole. The common types of metonymy in this video are the Individual Entity \& Whole and Piece of Clothing \& Person.

\subsection{Multi-model Metonymy of Individual Entity \& Whole}


The individual entity \& whole in metonymy can convey as much information as possible in a short duration of propaganda (Wang Xiaomei, 2019), which is fully utilized in the Graduation Answers, covering as many students as possible (as shown in Table 2). Tsinghua has about 3,400 freshmen learning different majors each year. In this video, 34 student representatives are selected to make full use of the mode of the individual entity $\&$ whole to convey the video theme to the audience.

There are three metonyms in the first part of the video. The freshman girl A who has no extracurricular interest refers to all students who lack extracurricular interest; The freshman boy B who lacks the sense of cooperation refers to all students who lack the sense of cooperation; The freshman girl $\mathrm{C}$ who is unconfident refers to the students who lack confidence. At the end of the first part of the video, the examiner went forward and told everyone: "Four years ago, you came to Tsinghua from all corners of the country, and different growth environments gave you different starting lines." The students appearing on the video represent the whole students of the same kind in the words of the examiner, which implement the cross-domain metonymy between picture modality and verbal symbol. In the second part of the video, there appear six metonymies. The decision-making girl D refers to all students who can decide their own life direction; The broad-minded boy $\mathrm{E}$ refers to all experienced graduates; The broad-minded boy E refers to all the experienced graduates; The boy with a personal interest refers to all the graduates who love what they choose; The boy $\mathrm{G}$ with cooperative spirit refers to all the graduates who have cooperative spirit; The girl H with self-improvement spirit refers to all graduates who have the spirit of self-improvement; The boy I with the dedication refers to all the dedicated graduates. At the end of the second part of the video, the examiner asked the last question: "In Tsinghua, have you become a better self?" The answer is yes. The students in the video represent all the graduations of Tsinghua, which also realizes the cross-domain metonymy of image symbols to verbal symbols.

\begin{tabular}{|c|c|}
\hline $\begin{array}{c}\text { Source domain } \\
\text { interest }\end{array}$ & Target domain \\
\hline $\begin{array}{c}\text { The freshman girls A who has no extracurricular } \\
\text { cooperation }\end{array}$ & All students who lack extracurricular interest \\
\hline The freshman boy B who lacks the sense of & All students who lack the sense of cooperation \\
\hline The freshman girl C who is unconfident & All students who lack confidence \\
\hline The decision-making girl D & All students who can decide their own life direction \\
\hline The broad-minded boy E & All experienced graduates \\
\hline The boy with a personal interest & All the graduates who love what they choose \\
\hline The boy G with a cooperative spirit & All the graduates who have a cooperative spirit \\
\hline The girl H with self-improvement spirit & All graduates who have the spirit of \\
& self-improvement \\
\hline The boy I with the dedication & All the dedicated graduates \\
\hline
\end{tabular}

Table 2 : Individual Entity \& Whole

\subsection{Multi-model Metonymy of Piece of Clothing \& Person}

The piece of clothing \& person in this video is also carefully processed. In the first part of the video, when the students first entered the school, every student's costume was a simple T-shirt with jeans, which is typical of a freshman or a lower grade student, and most of the footage of the video is on-campus life. Besides, the questions concerned of this period are all about the freshman (See Table 1 Part 1 for details), which realizes the multi-model metonymy between picture and verbal symbols. However, in the second part of the video, many students were on the job and gradually entered society. The environment in the video is no longer limited to the campus, but the graduation ceremony, overseas practice base, education mailbox and so on. 
Besides, the students are also wearing mature shirts with trousers or bachelor suits, which refers to graduates who are about to face jobs or have begun working. The questions here are all about the graduations of Tsinghua (See Table 1 Part 2 for details), which also achieves the cross-domain metonymy of picture symbols to verbal symbols.

\section{Conclusion}

"Graduation Answers" stresses the growth of students in the university in four years, highlighting the school spirit and strong cultural heritage of Tsinghua University through the two parts of the question comparison. The propaganda film uses multimodal metaphors such as images, words, gestures, music, etc. and metonymies, vividly showing the audience the unique culture of self-improvement and social commitment, which has aroused the audience's strong resonance. Different from other college propaganda films, the video does not directly introduce the equipment, teachers, and scientific research groups of the school. Instead, it displays the cultural heritage of Tsinghua in front of the audience through 13 questions and the design of the scene, which shows the audience a Tsinghua with a patriotic dedication and the pursuit of excellence, a university, which is rigorous in learning and honesty, inclusive and humanistic.

\section{References:}

[1] Dong Bo.The Expansion of the Influence of College Admissions Advertisements on University Culture[J].Today Mass Media,2019,27(01):131-133.

[2] Forceville C. Metaphor in Pictures and Multimodal Representations[J]. The Cambridge and Book of Metaphor and Thought, 2008: 462-482.

[3] Forceville C. Non-verbal and Multimodal Metaphor in a Cognitivist Framework: Agendas for Research[J]. Applications of Cognitive Linguistics, 2006, 1: 379.

[4] Forceville C. Pictorial metaphor in advertising [M]. Routledge, 2002.

[5] Guan Ke. Multimodal Metaphors in "Chinese Dream" Series of Public Service Advertisements[J]. Journal of Hunan University of Science \& Technology (Social Science Edition), 2015, 18(04): 156-161.

[6] Han Yafeng.Interactive Integration of Multimodal Metaphors and Metonymy in TV Public Service Advertisements[J].Journal of Henan Institute of Science and Technology, 2015(11):52-54.

[7] Lakoff G, Johnson M. Metaphors We Live by[M]. Chicago: University of Chicago Press, 1980.

[8] Liu Zhengguang.On the Continuum Relationship between Metonymy and Metaphor[J].Modern Foreign Languages, 2002(01):62-70+61.

[9] Pan Hongying. Cognitive Construction of Multimodal Metaphor in Advertisement for Public Good_-Based on the Series of Saving Paper from CCTV[J]. Journal of Hebei United University(Social Science Edition), 2013, 13(06): 128-130.

[10] Peirsman Y , Geeraerts D . Metonymy as a Prototypical Category [ J ].Cognitive Linguistics , 2006(03): $269-316$.

[11] Lakoff, G., Turner, M. More than cool reason: A Field Guide to Poetic metaphor[M] University of Chicago Press, 2009.

[12] Van Leeuwen T. Introducing Social Semiotics[M]. London: Routledge , 2005: 230.

[13] Xu Xiaoxin. Comparative Study of Multimodal Metaphors in American and Chinese Public Service Advertisements[D]. Qufu Normal University, 2013. 
[14] Yan Jia. Cognitive construction and co-occurrence of multimodal metaphor and metonymy in internet advertisement-a case study of Alipay advertisement [ J]. Journal of Shanxi Agricultural University(Social Science Edition), 2016(01): 63 - 69

[15] Yang Xu, Wang Shaohua. Analysis of Multimodal Metaphor Mechanism of TV Advertising Music[J]. Foreign Language and Literature Studies, 2013, 30(03): 160-165.

[16] Wang Xiaomei.Construction of Chinese National Image from the Perspective of multimodal Metaphor and Metonymy-A Case Study on China's One Minute[J].Journal of Xinyu University,2019,24(03):91-96.

[17] Wang Yang, Xiang Enbai.An Analysis of Multimodal Metaphorical Representation in Domestic and Foreign Automobile Print Advertisement[J].Foreign Language and Literature, 2016, 32(02): 85-92.

[18] Zhao Xiufeng.New Development of Conceptual Metaphor Studies: Multimodal Metaphor[J].Foreign Languages Research, 2011(01):1-10+112.

[19] Zhao Xiufeng, SuHuiyan. Cognitive Meaning Construction of Metaphorical Multimodal Discourse-Blending on the Interplay of Multimetonymy and Multimetaphor[J]. Journal of University of Science and Technology Beijing(Social Sciences Edition), 2010, 26(04): 18-24, 30.

[20] Zhang Hui, Zhan Weiwei.Dynamic Construction of Multimodal Metonymy and Metaphor in Advertising Discourse[J].Foreign Languages Research, 2011(01):16-23. 